\title{
Positive Solutions for the Initial Value Problems of Fractional Evolution Equation
}

\author{
Yue Liang, Yu Ma, and Xiaoyan Gao \\ Science College of Gansu Agricultural University, Lanzhou 730070, China \\ Correspondence should be addressed to Yue Liang; liangyuegsau@163.com
}

Received 27 July 2013; Accepted 22 September 2013

Academic Editor: S. A. Mohiuddine

Copyright (c) 2013 Yue Liang et al. This is an open access article distributed under the Creative Commons Attribution License, which permits unrestricted use, distribution, and reproduction in any medium, provided the original work is properly cited.

\begin{abstract}
This paper discusses the existence of positive solutions for the initial value problem of fractional evolution equation with noncompact semigroup $D^{q} u(t)+A u(t)=f(t, u(t)), t \geq 0 ; u(0)=u_{0}$ in a Banach space $X$, where $D^{q}$ denotes the Caputo fractional derivative of order $q \in(0,1), A: D(A) \subset X \rightarrow X$ is a closed linear operator, $-A$ generates an equicontinuous $C_{0}$ semigroup, and $f:[0, \infty) \times X \rightarrow X$ is continuous. In the case where $f$ satisfies a weaker measure of noncompactness condition and a weaker boundedness condition, the existence results of positive and saturated mild solutions are obtained. Particularly, an existence result without using measure of noncompactness condition is presented in ordered and weakly sequentially complete Banach spaces. These results are very convenient for application. As an example, we study the partial differential equation of parabolic type of fractional order.
\end{abstract}

\section{Introduction}

The theory of fractional differential equations is a new and important branch of differential equation theory, which has an extensive physical background and realistic mathematical model; see [1-6]. Correspondingly, the existence of solutions to fractional evolution equations in Banach space has also been studied by several authors; see [7-17]. In [7, 8], El-Borai first constructed the type of mild solutions to fractional evolution equations in terms of a probability density. And then they investigated the existence, uniqueness, and regularity of solutions to fractional integrodifferential equations in $[9,10]$. Recently, this theory was developed by Zhou et al. [11-14]. In [15-17], the authors studied the existence of mild solutions to fractional impulsive evolutions equations. But as far as we know, there are seldom results on the existence of positive solutions to the fractional evolution equations; see [18-20].

In this paper, we use the Sadovskii's fixed point theorem and monotone iterative technique to discuss the existence of positive and saturated mild solutions for the initial value problem (IVP) of fractional evolution equations:

$$
\begin{gathered}
D^{q} u(t)+A u(t)=f(t, u(t)), \quad t \geq 0, \\
u(0)=u_{0}
\end{gathered}
$$

in Banach space $X$, where $D^{q}$ denotes the Caputo fractional derivative of order $q \in(0,1), A: D(A) \subset X \rightarrow X$ is a closed linear operator, $-A$ generates a $C_{0}$-semigroup $S(t)(t \geq 0)$ in $X$, and $f: \mathbb{R}^{+} \times X \rightarrow X$ is continuous and will be specified later, $\mathbb{R}^{+}=[0, \infty)$.

In some existing articles, the fractional evolution equations were treated under the hypothesis that (I) $-A$ generates a compact semigroup or (II) the nonlinearity $f(t, u)$ is Lipschitz continuous in $u$ on a bounded set. For the case (I), the continuity of nonlinearity $f$ can guarantee the local existence of solutions. Hence it is convenient to apply to partial differential equations with compact resolvent. But for the case of noncompact semigroup, the condition (II) is not easy to verify sometimes. To make the things more applicable, in this work, we will prove the existence of mild solutions of the IVP(1) under the measure of noncompactness conditions. We will see that our conditions are weaker than the condition (II). In addition, we obtain the existence of positive mild solutions of the IVP(1) in this work, which is studied seldom before.

The rest of this paper is organized as follows. In Section 2, some preliminaries are given on the fractional calculus and the measure of noncompactness. In Section 3, we study the existence of positive and saturated mild solutions of the 
IVP(1). An example is given in Section 4 to illustrate the applicability of the abstract results obtained in Section 3.

\section{Preliminaries}

In this section, we introduce some basic facts about the fractional calculus and the measure of noncompactness that are used throughout this paper.

Let $X$ be a Banach space with norm $\|\cdot\|$, let $A: D(A) \subset$ $X \rightarrow X$ be a closed linear operator, and $-A$ generates a $C_{0^{-}}$ semigroup $S(t)(t \geq 0)$ in $X$. It is well known that there exist $\bar{M}>0$ and $\delta \in \mathbb{R}$ such that

$$
\|S(t)\| \leq \bar{M} e^{\delta t}, \quad t \geq 0 .
$$

Let $T>0$ be a constant. If $t \in[0, T]$, it follows from (2) that there exists a constant $M>0$ such that $\|S(t)\| \leq M$.

Let us recall the following known definitions in fractional calculus. For more details, see $[7,8,11-14,16,17]$ and the reference therein.

Definition 1. The fractional integral of order $\sigma>0$ with the lower limits zero for a function $f$ is defined by

$$
I^{\sigma} f(t)=\frac{1}{\Gamma(\sigma)} \int_{0}^{t}(t-s)^{\sigma-1} f(s) d s, \quad t>0,
$$

where $\Gamma$ is the gamma function.

The Riemann-Liouville fractional derivative of order $n-$ $1<\sigma<n$ with the lower limits zero for a function $f$ can be written as

$$
\begin{array}{r}
{ }^{L} D^{\sigma} f(t)=\frac{1}{\Gamma(n-\sigma)} \frac{d^{n}}{d t^{n}} \int_{0}^{t}(t-s)^{n-\sigma-1} f(s) d s \\
t>0, n \in \mathbb{N} .
\end{array}
$$

Also the Caputo fractional derivative of order $n-1<\sigma<n$ with the lower limits zero for a function $f \in C^{n}[0, \infty)$ can be written as

$$
\begin{array}{r}
D^{\sigma} f(t)=\frac{1}{\Gamma(n-\sigma)} \int_{0}^{t}(t-s)^{n-\sigma-1} f^{(n)}(s) d s, \\
t>0, n \in \mathbb{N} .
\end{array}
$$

Remark 2. (1) The Caputo derivative of a constant is equal to zero.

(2) If $f$ is an abstract function with values in $X$, then integrals which appear in Definition 1 are taken in Bochner's sense.

Lemma 3 (see [12]). A measurable function $h:[0, T] \rightarrow X$ is Bochner integrable if $\|h\|$ is Lebesgue integrable.

For $x \in X$, we define two families $\{U(t)\}_{t \geq 0}$ and $\{V(t)\}_{t \geq 0}$ of operators by

$$
\begin{gathered}
U(t) x=\int_{0}^{\infty} \eta_{q}(\theta) S\left(t^{q} \theta\right) x d \theta, \\
V(t) x=q \int_{0}^{\infty} \theta \eta_{q}(\theta) S\left(t^{q} \theta\right) x d \theta, \quad 0<q<1,
\end{gathered}
$$

where

$$
\begin{gathered}
\eta_{q}(\theta)=\frac{1}{q} \theta^{-1-(1 / q)} \rho_{q}\left(\theta^{-1 / q}\right), \\
\rho_{q}(\theta)=\frac{1}{\pi} \sum_{n=1}^{\infty}(-1)^{n-1} \theta^{-q n-1} \frac{\Gamma(n q+1)}{n !} \sin (n \pi q), \\
\theta \in(0, \infty),
\end{gathered}
$$

where $\eta_{q}$ is a probability density function defined on $(0, \infty)$, which has properties $\eta_{q}(\theta) \geq 0$ for all $\theta \in(0, \infty)$ and $\int_{0}^{\infty} \eta_{q}(\theta) d \theta=1$. Clearly, if the semigroup $S(t)(t \geq 0)$ is positive, then the operators $U(t)$ and $V(t)$ are also positive for all $t \geq 0$.

The following lemma is needed in the proof of the main results.

Lemma 4. The operators $U(t)$ and $V(t)$ have the following properties.

(i) For any fixed $t \geq 0$ and any $x \in X$, one has

$$
\begin{gathered}
\|U(t) x\| \leq M\|x\|, \\
\|V(t) x\| \leq \frac{q M}{\Gamma(1+q)}\|x\|=\frac{M}{\Gamma(q)}\|x\| .
\end{gathered}
$$

(ii) The operators $U(t)$ and $V(t)$ are strongly continuous for all $t \geq 0$.

(iii) If $S(t)(t \geq 0)$ is a equicontinuous semigroup, then $U(t)$ and $V(t)$ are equicontinuous in $X$ for $t>0$.

Proof. (i) and (ii) can be found in $[12,13]$, and we only need to prove (iii). For any $0 \leq t_{1}<t_{2} \leq T$, we have

$$
\begin{array}{r}
\left\|U\left(t_{2}\right)-U\left(t_{1}\right)\right\|=\int_{0}^{\infty} \eta_{q}(\theta)\left\|S\left(t_{2}^{q} \theta\right)-S\left(t_{1}^{q} \theta\right)\right\| d \theta, \\
\left\|V\left(t_{2}\right)-V\left(t_{1}\right)\right\|=q \int_{0}^{\infty} \theta \eta_{q}(\theta)\left\|S\left(t_{2}^{q} \theta\right)-S\left(t_{1}^{q} \theta\right)\right\| d \theta .
\end{array}
$$

According to the equicontinuity of $S(t)$ for $t>0$, we see that $\left\|U\left(t_{2}\right)-U\left(t_{1}\right)\right\|$ and $\left\|V\left(t_{2}\right)-V\left(t_{1}\right)\right\|$ tend to zero as $t_{2}-t_{1} \rightarrow 0$, which means that the operators $U(t)$ and $V(t)$ are equicontinuous in $X$ for $t>0$.

Let $t_{0} \geq 0$ be a constant and $I=\left[t_{0}, T\right]$. We denote by $C(I, X)$ the Banach space of all continuous $X$ value functions on interval $I$ with the norm $\|u\|_{C}=\max _{t \in I}\|u(t)\|$. Let $\alpha(B)$ denote the Kuratowski measure of noncompactness of the bounded set $B$ in $X$ and $C(I, X)$. It is clear that $0 \leq \alpha(B)<\infty$. If $\alpha(B)=0$, then the set $B$ is relatively compact. For more details of the definition and properties of the measure of noncompactness; see [21]. For any $B \subset C(I, X)$ and $t \in I$, set $B(t)=\{u(t): u \in B\} \subset X$. If $B$ is bounded in $C(I, X)$, then $B(t)$ is bounded in $X$, and $\alpha(B(t)) \leq \alpha(B)$. A mapping $Q: B \rightarrow B$ is said to be condensing if $\alpha(Q(B))<\alpha(B)$. The following Lemmas will be used in the proof of the main results. 
Lemma 5 (see $[22])$. Let $B \in C(I, X)$ be bounded and equicontinuous. Then $\alpha(B(t))$ is continuous on $I$ and

$$
\alpha(B)=\max _{t \in I} \alpha(B(t))=\alpha(B(I)),
$$

where $B(I)=\{x(t): x \in B, t \in I\}$.

Lemma 6 (see [23]). Let $B=\left\{u_{n}\right\} \subset C(I, X)$ be countable. If there exists $\psi \in L^{1}(I)$ such that $\left\|u_{n}(t)\right\| \leq \psi(t)$ a.e. $t \in I$, $n=1,2, \ldots$, then $\alpha(B(t))$ is Lebesgue integral on $I$ and

$$
\alpha\left(\left\{\int_{I} u_{n}(t) d t: n \in \mathbb{N}\right\}\right) \leq 2 \int_{I} \alpha(B(t)) d t
$$

Lemma 7 (see [24]). Let $B \subset C(I, X)$ be bounded. Then there exists a countable subset $B_{0}$ of $B$ such that $\alpha(B) \leq 2 \alpha\left(B_{0}\right)$.

Lemma 8 (see [25] (Sadovskii's fixed point theorem)). Let $X$ be a Banach space and let $\Omega$ be a nonempty bounded convex closed set in $X$. If $Q: \Omega \rightarrow \Omega$ is a condensing mapping, then $Q$ has a fixed point in $\Omega$.

In the proof of the main results, we also need the following generalized Gronwall-Bellman inequality, which can be found in [26, Page 188].

Lemma 9. Suppose $b \geq 0, \beta>0$, and $a(t)$ is a nonnegative function locally integrable on $0 \leq t<T$ (some $T \leq \infty$ ), and suppose $u(t)$ is nonnegative and locally integrable on $0 \leq t<T$ with

$$
u(t) \leq a(t)+b \int_{0}^{t}(t-s)^{\beta-1} u(s) d s
$$

on this interval, and then

$$
\begin{array}{r}
u(t) \leq a(t)+\int_{0}^{t}\left[\sum_{n=1}^{\infty} \frac{(b \Gamma(\beta))^{n}}{\Gamma(n \beta)}(t-s)^{n \beta-1} a(s)\right] d s \\
0 \leq t<T .
\end{array}
$$

Remark 10. In Lemma 9, if $a(t) \equiv 0$ for all $0 \leq t<T$, we easily see that $u(t)=0$.

For any $x_{0} \in X$ and $h \in C(I, X)$, a function $u$ is called the mild solution of the initial value problem

$$
\begin{aligned}
D^{q} u(t)+A u(t) & =h(t), \quad t \in I, \\
u\left(t_{0}\right) & =x_{0},
\end{aligned}
$$

if $u \in C(I, X)$ satisfies the integral equation:

$$
\begin{array}{r}
u(t)=U\left(t-t_{0}\right) x_{0}+\int_{t_{0}}^{t}(t-s)^{q-1} V(t-s) h(s) d s, \\
t \in I .
\end{array}
$$

Definition 11. By a mild solution of the $\operatorname{IVP}(1)$, we mean a function $u \in C(I, X)$ satisfying

$$
u(t)=U(t) u_{0}+\int_{0}^{t}(t-s)^{q-1} V(t-s) f(s, u(s)) d s
$$

for all $t \geq 0$.

\section{Existence of Positive Mild Solutions}

In this section, we introduce the existence theorems of positive mild solutions of the $\operatorname{IVP}(1)$. The discussions are based on fractional calculus and fixed point theorems.

Let $\lambda_{1}$ be the smallest positive real eigenvalue of the linear operator $A$, and let $e_{1} \in D(A)$ be the positive eigenvector corresponding to $\lambda_{1}$. Our main results are as follows.

Theorem 12. Let $X$ be a Banach space, let $A: D(A) \subset X \rightarrow$ $X$ be a closed linear operator, and $-A$ generate a positive and equicontinuous $C_{0}$-semigroup $S(t)(t \geq 0)$ in $X$. Assume that $f \in C\left(\mathbb{R}^{+} \times X, X\right)$ and, for any $T>0, f$ satisfies the following conditions.

$\left(H_{1}\right)$ There exist $a, b \in L^{1 / q_{1}}\left([0, T], \mathbb{R}^{+}\right), q_{1} \in(0, q)$ such that

$$
\|f(t, x)\| \leq a(t)\|x\|+b(t), \quad t \in[0, T], x \in X .
$$

$\left(H_{2}\right)$ For any $u \in C([0, T], X)$ with $u(t) \geq \sigma e_{1}, t \in[0, T]$, we have

$$
f(t, u(t)) \geq f\left(t, \sigma e_{1}\right), \quad t \in[0, T],
$$

where $\sigma>0$ is a constant.

$\left(\mathrm{H}_{3}\right)$ For any bounded set $\mathrm{D} \subset \mathrm{X}$, there exists a constant $L>0$ such that

$$
\alpha(f(t, D)) \leq L \alpha(D), \quad t \in[0, T] .
$$

If $f\left(t, \sigma e_{1}\right) \geq \lambda_{1} \sigma e_{1}$ and $u_{0} \in X$ with $u_{0} \geq \sigma e_{1}$, then the IVP(1) has at least one positive and saturated mild solution $u \in C([0, T), X)$. And if $T<\infty$, one has $\lim _{t \in T^{-}}\|u(t)\|=\infty$.

Proof. For any $t_{0} \geq 0$ and $x_{0} \in X$ with $x_{0} \geq \sigma e_{1}$, we first prove that there exists a constant $h_{t_{0}}=h\left(t_{0},\left\|x_{0}\right\|\right)>0$ such that the initial value problem (IVP)

$$
\begin{gathered}
D^{q} u(t)+A u(t)=f(t, u(t)), \quad t>t_{0}, \\
u\left(t_{0}\right)=x_{0}
\end{gathered}
$$

has at least one positive mild solution on $J=\left[t_{0}, t_{0}+h_{t_{0}}\right]$. For this purpose, we define an operator $Q$ by

$$
(Q u)(t)=U\left(t-t_{0}\right) x_{0}+\int_{t_{0}}^{t}(t-s)^{q-1} V(t-s) f(s, u(s)) d s
$$


Then $Q: C(J, X) \rightarrow C(J, X)$ is continuous, and the mild solutions of the IVP(20) are equivalent to the fixed point of the operator $Q$.

Let $R_{t_{0}}:=2 M\left(\left\|x_{0}\right\|+1\right)+\sigma e_{1}>0$. Denote

$\Omega_{R_{t_{0}}}:=\left\{u \in C(J, X):\|u(t)\| \leq R_{t_{0}}, u(t) \geq \sigma e_{1}, t \in J\right\}$.

Then $\Omega_{R_{t_{0}}} \subset C(J, X)$ is a nonempty bounded convex closed set. Let $h_{t_{0}}=h\left(t_{0},\left\|x_{0}\right\|\right)=\min \left\{1,\left(\Gamma(q)(1+c)^{1-q_{1}}\left(\left\|x_{0}\right\|+1\right) /\right.\right.$ $\left.\left.\left(R_{t_{0}} a_{0}+b_{0}\right)\right)^{1 /\left(q-q_{1}\right)},(\Gamma(q+1) /(4 M L+1))^{1 / q}\right\}$, where $c=$ $\left((q-1) /\left(1-q_{1}\right)\right) \in(-1,0), a_{0}=\|a\|_{L^{1 / q_{1}}\left(\left[t_{0}, t_{0}+1\right], \mathbb{R}^{+}\right)}, b_{0}=$ $\|b\|_{L^{1 / q_{1}}\left(\left[t_{0}, t_{0}+1\right], \mathbb{R}^{+}\right)}$. Then for any $u \in \Omega_{R_{t_{0}}}$ and $t \in J$, by Lemma 4(i), $\left(H_{1}\right)$, and (21), we have

$$
\begin{aligned}
& \|(Q u)(t)\| \\
& \leq\left\|U\left(t-t_{0}\right) x_{0}\right\| \\
& \quad+\int_{t_{0}}^{t}(t-s)^{q-1}\|V(t-s) f(s, u(s))\| d s \\
& \leq M\left\|x_{0}\right\|+\frac{M}{\Gamma(q)} \int_{t_{0}}^{t}(t-s)^{q-1}[a(s)\|u(s)\|+b(s)] d s \\
& =M\left\|x_{0}\right\|+\frac{M}{\Gamma(q)}\left[R_{t_{0}} \int_{t_{0}}^{t}(t-s)^{q-1} a(s) d s\right. \\
& \left.\quad+\int_{t_{0}}^{t}(t-s)^{q-1} b(s) d s\right] \\
& \leq M\left\|x_{0}\right\|+\frac{M\left(R_{t_{0}} a_{0}+b_{0}\right)}{\Gamma(q)(1+c)^{1-q_{1}}} \cdot h_{t_{0}}^{q-q_{1}} \leq R_{t_{0}} .
\end{aligned}
$$

Let $v_{0} \equiv \sigma e_{1}$. Then $v_{0}(t)=\sigma e_{1}$ for any $t \in J$ and

$$
\begin{array}{r}
\phi(t) \triangleq D^{q} v_{0}(t)+A v_{0}(t)=\lambda_{1} \sigma e_{1} \leq f\left(t, \sigma e_{1}\right), \\
t \in J .
\end{array}
$$

By the positivity of semigroup $S(t)(t \geq 0)$, the assumption $\left(H_{2}\right)$ and (21), for any $u \in \Omega_{R_{t_{0}}}$ and $t \in J$, we have

$$
\begin{aligned}
\sigma e_{1}= & v_{0}(t) \\
= & U\left(t-t_{0}\right) v_{0}\left(t_{0}\right) \\
& +\int_{t_{0}}^{t}(t-s)^{q-1} V(t-s) \phi(s) d s \\
\leq & U\left(t-t_{0}\right) \sigma e_{1} \\
& +\int_{t_{0}}^{t}(t-s)^{q-1} V(t-s) f\left(s, \sigma e_{1}\right) d s \\
\leq & U\left(t-t_{0}\right) x_{0} \\
& +\int_{t_{0}}^{t}(t-s)^{q-1} V(t-s) f(s, u(s)) d s \\
= & (Q u)(t) .
\end{aligned}
$$

Thus, $Q: \Omega_{R_{t_{0}}} \rightarrow \Omega_{R_{t_{0}}}$ is continuous and it implies that $\left(Q \sigma e_{1}\right)(t) \leq(Q u)(t)$ for any $u \in \Omega_{R_{t_{0}}}$ and $t \in J$.

Now, we prove that the set $Q\left(\Omega_{R_{t_{0}}}\right):=\left\{Q u: u \in \Omega_{R_{t_{0}}}\right\}$ is equicontinuous in $C(J, X)$. For any $u \in \Omega_{R_{t_{0}}}$ and $t_{0} \leq t_{1}<$ $t_{2} \leq t_{0}+h_{t_{0}}$, it follows from assumption $\left(H_{1}\right)$ and (21) that

$$
\begin{aligned}
& \left\|(Q u)\left(t_{2}\right)-(Q u)\left(t_{1}\right)\right\| \\
& \leq\left\|U\left(t_{2}-t_{0}\right) x_{0}-U\left(t_{1}-t_{0}\right) x_{0}\right\| \\
& +\int_{t_{1}}^{t_{2}}\left(t_{2}-s\right)^{q-1} \\
& \times\left\|V\left(t_{2}-s\right) f(s, u(s))\right\| d s \\
& +\int_{t_{0}}^{t_{1}}\left|\left(t_{2}-s\right)^{q-1}-\left(t_{1}-s\right)^{q-1}\right| \\
& \cdot\left\|V\left(t_{2}-s\right) f(s, u(s))\right\| d s \\
& +\int_{t_{0}}^{t_{1}}\left(t_{1}-s\right)^{q-1} \\
& \times \|\left[V\left(t_{2}-s\right)-V\left(t_{1}-s\right)\right] \\
& \times f(s, u(s)) \| d s \\
& \leq M\left\|U\left(t_{2}-t_{1}\right) x_{0}-x_{0}\right\|+\frac{M}{\Gamma(q)} \int_{t_{1}}^{t_{2}}\left(t_{2}-s\right)^{q-1} \\
& \times[a(s)\|u(s)\|+b(s)] d s \\
& +\frac{M}{\Gamma(q)} \int_{t_{0}}^{t_{1}} \mid\left(t_{2}-s\right)^{q-1} \\
& -\left(t_{1}-s\right)^{q-1} \\
& \cdot[a(s)\|u(s)\|+b(s)] d s \\
& +\int_{t_{0}}^{t_{1}}\left(t_{1}-s\right)^{q-1} \\
& \times \|\left[V\left(t_{2}-s\right)-V\left(t_{1}-s\right)\right] \\
& \times f(s, u(s)) \| d s \\
& \triangleq I_{1}+I_{2}+I_{3}+I_{4} \text {. }
\end{aligned}
$$

By Lemma 4(ii), it is easy to see that $I_{1} \rightarrow 0$ independently of $u \in \Omega_{R_{t_{0}}}$ as $t_{2}-t_{1} \rightarrow 0$ :

$$
\begin{gathered}
I_{2} \leq \frac{M}{\Gamma(q)}\left[R_{t_{0}} \int_{t_{1}}^{t_{2}}\left(t_{2}-s\right)^{q-1} a(s) d s\right. \\
\left.\quad+\int_{t_{1}}^{t_{2}}\left(t_{2}-s\right)^{q-1} b(s) d s\right] \\
\leq \frac{M\left(R_{t_{0}} a_{0}+b_{0}\right)}{\Gamma(q)(1+c)^{1-q_{1}}}\left(t_{2}-t_{1}\right)^{q-q_{1}} .
\end{gathered}
$$


Hence $I_{2} \rightarrow 0$ independently of $u \in \Omega_{R_{t_{0}}}$ as $t_{2}-t_{1} \rightarrow 0$ :

$$
\begin{gathered}
I_{3} \leq \frac{M}{\Gamma(q)}\left[R_{t_{0}} \int_{t_{0}}^{t_{1}}\left|\left(t_{2}-s\right)^{q-1}-\left(t_{1}-s\right)^{q-1}\right| \times a(s) d s\right. \\
\left.\quad+\int_{t_{0}}^{t_{1}}\left|\left(t_{2}-s\right)^{q-1}-\left(t_{1}-s\right)^{q-1}\right| b(s) d s\right] \\
\leq \frac{M}{\Gamma(q)}\left[R _ { t _ { 0 } } \left(\int_{t_{0}}^{t_{1}} \mid\left(t_{2}-s\right)^{q-1}\right.\right. \\
\left.-\left.\left(t_{1}-s\right)^{q-1}\right|^{1 /\left(1-q_{1}\right)} d s\right)^{1-q_{1}}
\end{gathered}
$$

$\cdot\|a\|_{L^{1 / q_{1}}\left(\left[t_{0} \cdot t_{1}\right], \mathbb{R}^{+}\right)}$

$+\left(\int_{t_{0}}^{t_{1}}\left|\left(t_{2}-s\right)^{q-1}-\left(t_{1}-s\right)^{q-1}\right|^{1 /\left(1-q_{1}\right)} d s\right)^{1-q_{1}}$

$\left.\cdot\|b\|_{\left.L^{1 / q_{1}\left(\left[t_{0} \cdot t_{1}\right], \mathbb{R}^{+}\right)}\right]}\right]$

$\leq \frac{M\left(R_{t_{0}} a_{0}+b_{0}\right)}{\Gamma(q)}$

$\times\left(\int_{t_{0}}^{t_{1}}\left[\left(t_{1}-s\right)^{c}-\left(t_{2}-s\right)^{c}\right] d s\right)^{1-q_{1}}$

$=\frac{M\left(R_{t_{0}} a_{0}+b_{0}\right)}{\Gamma(q)(1+c)^{1-q_{1}}}$

$\times\left(\left(t_{1}-t_{0}\right)^{1+c}-\left(t_{2}-t_{0}\right)^{1+c}+\left(t_{2}-t_{1}\right)^{1+c}\right)^{1-q_{1}}$

$\leq \frac{M\left(R_{t_{0}} a_{0}+b_{0}\right)}{\Gamma(q)(1+c)^{1-q_{1}}}\left(t_{2}-t_{1}\right)^{q-q_{1}}$.

It follows that $I_{3} \rightarrow 0$ independently of $u \in \Omega_{R_{t_{0}}}$ as $t_{2}-t_{1} \rightarrow$ 0 . For $t_{1}=t_{0}, t_{0}<t_{2} \leq t_{0}+h_{t_{0}}$, it is easy to see that $I_{4}=0$. Let $t_{1}>t_{0}$ and $\epsilon \in\left(0, t_{1}-t_{0}\right)$ be small enough, and we have

$$
\begin{gathered}
I_{4} \leq \int_{t_{0}}^{t_{1}-\epsilon}\left(t_{1}-s\right)^{q-1} \|\left[V\left(t_{2}-s\right)-V\left(t_{1}-s\right)\right] \\
\times f(s, u(s)) \| d s \\
+\int_{t_{1}-\epsilon}^{t_{1}}\left(t_{1}-s\right)^{q-1} \|\left[V\left(t_{2}-s\right)-V\left(t_{1}-s\right)\right] \\
\times f(s, u(s)) \| d s \\
\leq \int_{t_{0}}^{t_{1}-\epsilon}\left(t_{1}-s\right)^{q-1}\left\|V\left(t_{2}-s\right)-V\left(t_{1}-s\right)\right\| \\
\cdot\|f(s, u(s))\| d s \\
+\frac{2 M}{\Gamma(q)} \int_{t_{1}-\epsilon}^{t_{1}}\left(t_{1}-s\right)^{q-1}\|f(s, u(s))\| d s
\end{gathered}
$$

$$
\begin{aligned}
& \leq \int_{t_{0}}^{t_{1}-\epsilon}\left(t_{1}-s\right)^{q-1}[a(s)\|u(s)\|+b(s)] d s \\
& \cdot \sup _{s \in\left[t_{0}, t_{1}-\epsilon\right]}\left\|V\left(t_{2}-s\right)-V\left(t_{1}-s\right)\right\| \\
& +\frac{2 M}{\Gamma(q)} \int_{t_{1}-\epsilon}^{t_{1}}\left(t_{1}-s\right)^{q-1} \\
& \quad \times[a(s)\|u(s)\|+b(s)] d s \\
& \leq \frac{R_{t_{0}} a_{0}+b_{0}}{(1+c)^{1-q_{1}}}\left(\left(t_{1}-t_{0}\right)^{1+c}-\epsilon^{1+c}\right)^{1-q_{1}} \\
& \cdot \sup _{s \in\left[t_{0}, t_{1}-\epsilon\right]}\left\|V\left(t_{2}-s\right)-V\left(t_{1}-s\right)\right\| \\
& +\frac{2 M\left(R_{t_{0}} a_{0}+b_{0}\right)}{\Gamma(q)(1+c)^{1-q_{1}}} \epsilon^{q-q_{1}} .
\end{aligned}
$$

Since Lemma 4(iii) implies the continuity of $V(t)$ for $t>0$ in the uniform operator topology, it is easy to see that $I_{4} \rightarrow 0$ independently of $u \in \Omega_{R_{t_{0}}}$ as $t_{2}-t_{1} \rightarrow 0$ and $\epsilon \rightarrow 0$. Thus, $\left\|(Q u)\left(t_{2}\right)-(Q u)\left(t_{1}\right)\right\| \rightarrow 0$ independently of $u \in \Omega_{R_{t_{0}}}$ as $t_{2}-$ $t_{1} \rightarrow 0$, which means that the $\operatorname{set} Q\left(\Omega_{R_{t_{0}}}\right)$ is equicontinuous.

It remains to prove that $Q: \Omega_{R_{t_{0}}} \rightarrow \Omega_{R_{t_{0}}}$ is a condensing mapping. Let $B \subset \Omega_{R_{t_{0}}}$ be a bounded set. By Lemma 7, there exists $B_{0}=\left\{u_{n}\right\} \subset B$ such that $\alpha(Q(B)) \leq 2 \alpha\left(Q\left(B_{0}\right)\right)$. Since $Q\left(B_{0}\right) \subset Q\left(\Omega_{R_{t_{0}}}\right) \subset \Omega_{R_{t_{0}}}$ is bounded and equicontinuous, by Lemma 5, it follows that $\alpha\left(Q\left(B_{0}\right)\right)=\max _{t \in J} \alpha\left(Q\left(B_{0}\right)(t)\right)$. Thus, for any $t \in J$, by (21), one has

$$
\begin{aligned}
\alpha\left(Q\left(B_{0}\right)(t)\right)=\alpha\left(\left\{U\left(t-t_{0}\right) x_{0}\right.\right. \\
\quad+\int_{t_{0}}^{t}(t-s)^{q-1} V(t-s) \\
\left.\left.\quad \times f\left(s, u_{n}(s)\right) d s: n \in \mathbb{N}\right\}\right) \\
=\alpha\left(\left\{\int_{t_{0}}^{t}(t-s)^{q-1} V(t-s)\right.\right. \\
\left.\left.\quad \times f\left(s, u_{n}(s)\right) d s: n \in \mathbb{N}\right\}\right) \\
\leq 2 \int_{t_{0}}^{t}(t-s)^{q-1}\|V(t-s)\| \\
\quad \cdot \alpha\left(f\left(s, B_{0}(s)\right)\right) d s \\
\leq \frac{2 q M L}{\Gamma(q+1)} \int_{t_{0}}^{t}(t-s)^{q-1} \alpha\left(B_{0}(s)\right) d s \\
=\frac{2 M L h_{t_{0}}^{q}}{\Gamma(q+1)} \alpha\left(B_{0}\right) \leq \frac{2 M L h_{t_{0}}^{q}}{\Gamma(q+1)} \alpha(B) .
\end{aligned}
$$


Thus, $\alpha(Q(B)) \leq 2 \alpha\left(Q\left(B_{0}\right)\right)=2 \max _{t \in J} \alpha\left(Q\left(B_{0}\right)(t)\right) \leq$ $\left(4 M L h_{t_{0}}^{q} / \Gamma(q+1)\right) \alpha(B)$, which means that $Q: \Omega_{R_{t_{0}}} \rightarrow \Omega_{R_{t_{0}}}$ is a condensing mapping. By Lemma 8 , the operator $Q$ has at least one fixed point $u^{*}$ in $\Omega_{R_{t_{0}}}$, and $u^{*}(t) \geq \sigma e_{1}>0$ for all $t \in J$. Hence $u^{*} \in C(J, X)$ is a positive mild solution of the $\operatorname{IVP}(20)$.

Hence, for the $\operatorname{IVP}(1)$, there exists an interval $\left[0, h_{0}\right]$ such that the $\operatorname{IVP}(1)$ has a positive mild solution $u$ on $\left[0, h_{0}\right]$. Now, by the extension theorem of initial value problem, $u$ can be extended to a saturated solution $u \in C([0, T), X)$ of the $\operatorname{IVP}(1)$, whose existence interval is $[0, T)$, and if $T<\infty$, one has $\lim _{t \rightarrow T^{-}}\|u(t)\|=\infty$.

For any $T>0$ and $r>0$, define a set $\Omega_{r}$ by

$$
\begin{aligned}
& \Omega_{r}=\{u \in C([0, T], X):\|u(t)\| \\
&\left.\leq r, u(t) \geq \sigma e_{1}, t \in[0, T]\right\} .
\end{aligned}
$$

If $f(t, u)$ is increasing in $\Omega_{r}$, that is, $f(t, u)$ satisfies the condition:

$\left(H_{4}\right)$ for any $u_{1}, u_{2} \in \Omega_{r}$ with $u_{1}(t) \leq u_{2}(t), t \in[0, T]$, we have

$$
f\left(t, u_{1}(t)\right) \leq f\left(t, u_{2}(t)\right), \quad t \in[0, T],
$$

then we have $f(t, u(t)) \geq f\left(t, \sigma e_{1}\right)$ for any $u \in \Omega_{r}$ and $t \in$ $[0, T]$. Hence by Theorem 12 , we have the following existence result.

Corollary 13. Let $X$ be a Banach space, let $A: D(A) \subset X \rightarrow$ $X$ be a closed linear operator, and $-A$ generates a positive and equicontinuous $C_{0}$-semigroup $S(t)(t \geq 0)$ in $X$. Assume that $f \in C\left(\mathbb{R}^{+} \times X, X\right)$ and, for any $T>0, f$ satisfies the conditions $\left(H_{1}\right),\left(H_{3}\right)$, and $\left(H_{4}\right)$. If $f\left(t, \sigma e_{1}\right) \geq \lambda_{1} \sigma e_{1}$ and $u_{0} \in X$ with $u_{0} \geq \sigma e_{1}$, then the IVP(1) has at least one positive and saturated mild solution $u \in C([0, T), X)$. And if $T<\infty$, one has $\lim _{t \in T^{-}}\|u(t)\|=\infty$.

Noticing that the condition $\left(\mathrm{H}_{3}\right)$ is not easy to verify in applications, we can weaken or delete the condition $\left(\mathrm{H}_{3}\right)$ in ordered Banach space.

Theorem 14. Let $X$ be an ordered Banach space, whose positive cone $K$ is normal, let $A: D(A) \subset X \rightarrow X$ be a closed linear operator, and $-A$ generates a positive and equicontinuous $C_{0^{-}}$ semigroup $S(t)(t \geq 0)$ in $X$. Assume that $f \in C\left(\mathbb{R}^{+} \times K, X\right)$ and for any $T>0, f$ satisfies the conditions $\left(H_{1}\right),\left(H_{4}\right)$, and

$\left(H_{5}\right)$ there exists a constant $L_{1}>0$ such that

$$
\alpha(f(t, D(t))) \leq L_{1} \alpha(D(t)), \quad t \in[0, T]
$$

for any increasing sequence $D=\left\{x_{n}\right\} \subset \Omega_{r}$.

If $f\left(t, \sigma e_{1}\right) \geq \lambda_{1} \sigma e_{1}$ and $u_{0} \in X$ with $u_{0} \geq \sigma e_{1}$, then the IVP(1) has at least one positive and saturated mild solution $u \in C([0, T), K)$. And if $T<\infty$, one has $\lim _{t \in T^{-}}\|u(t)\|=\infty$.
Proof. For any $t_{0} \geq 0$ and $x_{0} \in X$ with $x_{0} \geq \sigma e_{1}$, we first prove that the $\operatorname{IVP}(20)$ has at least one positive mild solution on $J=\left[t_{0}, t_{0}+h_{t_{0}}\right]$, where $h_{t_{0}}=\min \{1,(\Gamma(q)(1+$ c) $\left.\left.{ }^{1-q_{1}}\left(\left\|x_{0}\right\|+1\right) /\left(R_{t_{0}} a_{0}+b_{0}\right)\right)^{1 /\left(q-q_{1}\right)}\right\}$. Define an operator $Q$ as in (21). Let $R_{t_{0}}=2 M\left(\left\|X_{0}\right\|+1\right)+\sigma e_{1}$. Write $\Omega_{R_{t_{0}}}$ as in (22). A similar argument as in the proof of Theorem 12 shows that $Q: \Omega_{R_{t_{0}}} \rightarrow \Omega_{R_{t_{0}}}$ is continuous and the set $Q\left(\Omega_{R_{t_{0}}}\right)$ is equicontinuous. From the assumption $\left(H_{4}\right)$, it is easy to see that $Q: \Omega_{R_{t_{0}}} \rightarrow \Omega_{R_{t_{0}}}$ is an increasing operator.

Let $v_{0} \equiv \sigma e_{1} \in \Omega_{R_{t_{0}}}$. Define a sequence $\left\{v_{n}\right\}$ by the iterative scheme

$$
v_{n}=Q v_{n-1}, \quad n=1,2, \ldots
$$

Since $v_{0}=\sigma e_{1} \leq Q\left(\sigma e_{1}\right)=Q\left(v_{0}\right)=v_{1}$, by the increasing property of the operator $Q$, we have

$$
v_{0} \leq v_{1} \leq v_{2} \leq \cdots \leq v_{n} \leq \cdots .
$$

By the equicontinuity property of the set $Q\left(\Omega_{R_{t_{0}}}\right)$, the set $\left\{v_{n}\right\}=\left\{Q v_{n-1}\right\} \subset Q\left(\Omega_{R_{t_{0}}}\right)$ is equicontinuous. Next, we prove that the set $\left\{v_{n}\right\}$ is uniformly convergent on $J$.

For convenience, let $B=\left\{v_{n}: n \in \mathbb{N}\right\}$ and $B_{0}=\left\{v_{n-1}\right.$ : $n \in \mathbb{N}\}$. From $B_{0}=B \cup\left\{v_{0}\right\}$, it follows that $\alpha\left(B_{0}(t)\right)=\alpha(B(t))$ for any $t \in J$. Let $\varphi(t):=\alpha(B(t))=\alpha\left(B_{0}(t)\right)$. By Lemma 6, assumption $\left(H_{5}\right)$, and (21), we have

$$
\begin{aligned}
& \varphi(t)= \alpha(B(t)) \\
&= \alpha\left(\left\{U\left(t-t_{0}\right) x_{0}+\int_{t_{0}}^{t}(t-s)^{q-1} V(t-s)\right.\right. \\
&\left.\left.\quad \times f\left(s, v_{n-1}(s)\right) d s: n \in \mathbb{N}\right\}\right) \\
&= \alpha\left(\left\{\int_{t_{0}}^{t}(t-s)^{q-1} V(t-s)\right.\right. \\
&\left.\left.\quad \times f^{2}\left(s, v_{n-1}(s)\right) d s: n \in \mathbb{N}\right\}\right) \\
& \leq 2 \int_{t_{0}}^{t}(t-s)^{q-1}\|V(t-s)\| \\
& \cdot \alpha\left(f\left(s, B_{0}(s)\right)\right) d s \\
& \leq \frac{2 M L_{1}}{\Gamma(q)} \int_{t_{0}}^{t}(t-s)^{q-1} \alpha\left(B_{0}(s)\right) d s \\
&=\frac{2 M L_{1}}{\Gamma(q)} \int_{t_{0}}^{t}(t-s)^{q-1} \varphi(s) d s .
\end{aligned}
$$

Hence by Lemma 9, $\alpha(B(t))=\varphi(t) \equiv 0$ for any $t \in J$. By Lemma 5, $\alpha(B)=\max _{t \in J} \alpha(B(t))=0$, from which we obtain that the set $\left\{v_{n}\right\}$ is relatively compact. Thus, there is a subset $\left\{v_{n_{k}}\right\} \subset\left\{v_{n}\right\}$ such that $v_{n_{k}} \rightarrow u^{*} \in \Omega_{R_{t_{0}}}$. Combining this with the monotonicity (35), we easily prove that $\left\{v_{n}\right\}$ itself is convergent in $\Omega_{R_{t_{0}}}$, that is, $v_{n} \rightarrow u^{*} \in \Omega_{R_{t_{0}}}$ as $n \rightarrow \infty$. 
Letting $n \rightarrow \infty$ in (34), by the continuity of the operator $Q$, we have $u^{*}=Q u^{*}$ and $u^{*}(t) \geq \sigma e_{1}>0$ for all $t \in J$. Hence $u^{*} \in C(J, X)$ is a positive mild solution of the $\operatorname{IVP}(20)$.

Hence, for the IVP(1), there exists an interval $\left[0, h_{0}\right]$ such that the IVP(1) has a positive mild solution $u$ on $\left[0, h_{0}\right]$. By the extension theorem of the initial value problem, $u$ can be extended to a saturated solution $u \in C([0, T), X)$ of the $\operatorname{IVP}(1)$, whose existence interval is $[0, T)$, and if $t<\infty$, then $\lim _{t \rightarrow T^{-}}\|u(t)\|=\infty$.

In Theorem 14, if $X$ is weakly sequentially complete, the condition $\left(\mathrm{H}_{5}\right)$ holds automatically. In fact, by [27, Theorem 2.2], any monotonic and order-bounded sequence is precompact. Let $D=\left\{x_{n}\right\} \subset \Omega_{r}$ be an increasing sequence. Then by the conditions $\left(H_{1}\right)$ and $\left(H_{4}\right),\left\{f\left(t, x_{n}\right)\right\}$ is a monotonic increasing and order-bounded sequence. By the property of the measure of noncompactness, we have

$$
\alpha\left(\left\{f\left(t, x_{n}\right)\right\}\right)=0 .
$$

Thus, the condition $\left(\mathrm{H}_{5}\right)$ holds. From Theorem 14, we have the following.

Corollary 15. Let $X$ be an ordered and weakly sequentially complete Banach space, whose positive cone $K$ is normal, let $A: D(A) \subset X \rightarrow X$ be a closed linear operator, and $-A$ generates a positive and equicontinuous $C_{0}$-semigroup $S(t)$ $(t \geq 0)$ in $X$. Assume that $f \in C\left(\mathbb{R}^{+} \times K, X\right)$ and, for any $T>0$, $f$ satisfies the conditions $\left(H_{1}\right)$ and $\left(H_{4}\right)$. If $f\left(t, \sigma e_{1}\right) \geq \lambda_{1} \sigma e_{1}$ and $u_{0} \in X$ with $u_{0} \geq \sigma e_{1}$, then the IVP(1) has at least one positive and saturated mild solution $u \in C([0, T), K)$. And if $T<\infty$, one has $\lim _{t \in T^{-}}\|u(t)\|=\infty$.

\section{Positive Mild Solutions of Parabolic Equations}

Let $\Omega \subset \mathbb{R}^{N}$ be a bounded domain with a sufficiently smooth boundary $\partial \Omega, F: \bar{\Omega} \times \mathbb{R}^{+} \times \mathbb{R} \rightarrow \mathbb{R}$. We consider the following problem of parabolic type:

$$
\begin{gathered}
\frac{\partial^{q}}{\partial t^{q}} u(x, t)+\Delta u(x, t)=F(x, t, u(x, t)) \\
\text { in } \Omega \times \mathbb{R}^{+}, \\
\left.u\right|_{\partial \Omega}=0, \\
u(x, 0)=\varphi(x) \quad \text { in } \Omega,
\end{gathered}
$$

where $0<q<1$ is a constant, and $\Delta$ is the Laplace operator. Let $X:=L^{2}(\Omega)$. Then $X$ is an ordered Banach space with the norm $\|f\|_{2}=\left(\int_{\Omega}|f(x)|^{2} d x\right)^{1 / 2}$ for any $f \in X$ and the partial order " $\leq$ ". $K:=\{u \in X: u(x) \geq 0$ a.e. $x \in \Omega\}$ is the positive cone in $X$. Consider the operator $A: D(A) \subset X \rightarrow X$ defined by

$$
D(A)=H^{2}(\Omega) \cap H_{0}^{1}(\Omega), \quad A u=-\Delta u .
$$

Then $-A$ generates a positive and analytic semigroup $S(t)$ $(t \geq 0)$ in $X$ (see $[28,29])$. Let $\lambda_{1}$ be the smallest positive real eigenvalue of operator $A$ under the Dirichlet boundary condition $\left.u\right|_{\partial \Omega}=0$ and let $e_{1}(x)$ be the positive eigenvector corresponding to $\lambda_{1}$. Then $\lambda_{1}>0$ and $e_{1}(x)>0$ for $x \in \Omega$. For any $T>0$ and $r>0$, denote by

$$
\begin{aligned}
P_{r}=\{u & \in C\left([0, T], L^{2}(\Omega)\right):\|u(x, t)\|_{2} \\
& \left.\leq r, u(x, t) \geq \sigma e_{1}(x), t \in[0, T]\right\},
\end{aligned}
$$

where $\sigma>0$ is a constant. Assume that $F: \bar{\Omega} \times \mathbb{R}^{+} \times \mathbb{R} \rightarrow \mathbb{R}$ is continuous with $F\left(x, t, \sigma e_{1}(x)\right) \geq \lambda_{1} \sigma e_{1}(x), x \in \Omega, t \in \mathbb{R}^{+}$ and satisfies the following conditions.

$\left(F_{1}\right)$ There exist $a, b \in L^{1 / q_{1}}\left([0, T], \mathbb{R}^{+}\right), q_{1} \in(0, q)$ such that

$$
\begin{array}{r}
|F(x, t, u(x, t))| \leq a(t)|u(x, t)|+b(t), \\
x \in \bar{\Omega}, t \in[0, T] .
\end{array}
$$

$\left(F_{2}\right)$ For any $u_{1}, u_{2} \in P_{r}$ with $u_{1} \leq u_{2}$, we have

$$
\begin{array}{r}
F\left(x, t, u_{1}(x, t)\right) \leq F\left(x, t, u_{2}(x, t)\right), \\
x \in \bar{\Omega}, \quad t \in[0, T] .
\end{array}
$$

Let $f: \mathbb{R}^{+} \times X \rightarrow X$ be defined by $f(t, u(t))(\cdot)=$ $F(\cdot, t, u(\cdot, t))$. Then $f \in C\left(\mathbb{R}^{+} \times X, X\right)$ with $f\left(t, \sigma e_{1}\right) \geq \lambda_{1} \sigma e_{1}$ for $t \in \mathbb{R}^{+}$and satisfies the assumptions $\left(H_{1}\right)$ and $\left(H_{4}\right)$. Therefore, by Corollary 15, we have the following existence result for the problem (38).

Theorem 16. Assume that $F \in C\left(\bar{\Omega} \times \mathbb{R}^{+} \times \mathbb{R}, \mathbb{R}\right)$ with $F\left(x, t, \sigma e_{1}(x)\right) \geq \lambda_{1} \sigma e_{1}(x)$ for $x \in \Omega, t \in \mathbb{R}^{+}$and satisfies the assumptions $\left(F_{1}\right)$ and $\left(F_{2}\right)$. If $\varphi \in C(\bar{\Omega})$ with $\varphi(x) \geq \sigma e_{1}(x)$ for any $x \in \bar{\Omega}$, then the problem (38) has at least one positive mild solution $u$, satisfies $u(x, t) \geq \sigma e_{1}(x)$ for any $x \in \bar{\Omega}$ and $t \in[0, T]$. And if $T<+\infty$, one has $\lim _{t \rightarrow T^{-}}|u(t)|=+\infty$.

Remark 17. In Theorem 16, we do not use the property of compactness of the semigroup $S(t)(t \geq 0)$, which is a key assumption in $[7-9,11,12]$.

\section{References}

[1] K. S. Miller and B. Ross, An Introduction to the Fractional Calculus and Fractional Differential Equations, A Wiley-Interscience Publication, John Wiley \& Sons, New York, NY, USA, 1993.

[2] I. Podlubny, Fractional Differential Equations, vol. 198 of Mathematics in Science and Engineering, Academic Press, San Diego, Calif, USA, 1999.

[3] A. A. Kilbas, H. M. Srivastava, and J. J. Trujillo, Theory and Applications of Fractional Differential Equations, vol. 204 of North-Holland Mathematics Studies, Elsevier Science, Amsterdam, The Netherlands, 2006.

[4] V. Lakshmikantham, S. Leela, and J. Devi, Theory of Fractional Dynamic Systems, Cambridge Scientific Publishers, Cambridge, UK, 2009.

[5] K. Diethelm, The Analysis of Fractional Differential Equations, vol. 2004 of Lecture Notes in Mathematics, Springer, Berlin, Germany, 2010. 
[6] R. P. Agarwal, V. Lakshmikantham, and J. J. Nieto, "On the concept of solution for fractional differential equations with uncertainty," Nonlinear Analysis: Theory, Methods \& Applications, vol. 72, no. 6, pp. 2859-2862, 2010.

[7] M. M. El-Borai, "Some probability densities and fundamental solutions of fractional evolution equations," Chaos, Solitons \& Fractals, vol. 14, no. 3, pp. 433-440, 2002.

[8] M. M. El-Borai, "The fundamental solutions for fractional evolution equations of parabolic type," Journal of Applied Mathematics and Stochastic Analysis, no. 3, pp. 197-211, 2004.

[9] M. M. El-Borai, "Semigroups and some nonlinear fractional differential equations," Applied Mathematics and Computation, vol. 149, no. 3, pp. 823-831, 2004.

[10] M. El-Borai, K. El-Nadi, and E. El-Akabawy, "Fractional evolution equations with nonlocal conditions," Journal of Applied Mathematics and Mechanics, vol. 4, no. 6, pp. 1-12, 2008.

[11] Y. Zhou and F. Jiao, "Nonlocal Cauchy problem for fractional evolution equations," Nonlinear Analysis: Real World Applications, vol. 11, no. 5, pp. 4465-4475, 2010.

[12] J. Wang and Y. Zhou, "A class of fractional evolution equations and optimal controls," Nonlinear Analysis: Real World Applications, vol. 12, no. 1, pp. 262-272, 2011.

[13] Y. Zhou and F. Jiao, "Existence of mild solutions for fractional neutral evolution equations," Computers \& Mathematics with Applications, vol. 59, no. 3, pp. 1063-1077, 2010.

[14] J. Wang, Y. Zhou, and W. Wei, "A class of fractional delay nonlinear integrodifferential controlled systems in Banach spaces," Communications in Nonlinear Science and Numerical Simulation, vol. 16, no. 10, pp. 4049-4059, 2011.

[15] Z. Tai and X. Wang, "Controllability of fractional-order impulsive neutral functional infinite delay integrodifferential systems in Banach spaces," Applied Mathematics Letters, vol. 22, no. 11, pp. 1760-1765, 2009.

[16] A. Debbouche and D. Baleanu, "Controllability of fractional evolution nonlocal impulsive quasilinear delay integrodifferential systems," Computers \& Mathematics with Applications, vol. 62, no. 3, pp. 1442-1450, 2011.

[17] G. M. Mophou, "Existence and uniqueness of mild solutions to impulsive fractional differential equations," Nonlinear Analysis: Theory, Methods \& Applications, vol. 72, no. 3-4, pp. 1604-1615, 2010.

[18] N. Nyamoradi, D. Baleanu, and T. Bashiri, "Positive solutions to fractional boundary value problems with nonlinear boundary conditions," Abstract and Applied Analysis, vol. 2013, Article ID 579740, 20 pages, 2013.

[19] D. Baleanu, H. Mohammadi, and Sh. Rezapour, "Positive solutions of an initial value problem for nonlinear fractional differential equations," Abstract and Applied Analysis, vol. 2012, Article ID 837437, 7 pages, 2012.

[20] H. Yang and Y. Liang, "Positive solutions for the initial value problem of fractional evolution equations," Abstract and Applied Analysis, vol. 2013, Article ID 428793, 7 pages, 2013.

[21] K. Deimling, Nonlinear Functional Analysis, Springer, Berlin, Germany, 1985.

[22] D. Guo and J. Sun, Ordinary Differential Equations in Abstract Spaces, Shandong Science and Technology, Jinan, China, 1989.

[23] H.-P. Heinz, "On the behaviour of measures of noncompactness with respect to differentiation and integration of vector-valued functions," Nonlinear Analysis: Theory, Methods \& Applications, vol. 7, no. 12, pp. 1351-1371, 1983.
[24] Y. X. Li, "Existence of solutions to initial value problems for abstract semilinear evolution equations," Acta Mathematica Sinica, vol. 48, no. 6, pp. 1089-1094, 2005.

[25] B. N. Sadovskiŭ, "On a fixed point principle," Funkcional'nyi Analiz i ego Priloženija, vol. 1, no. 2, pp. 74-76, 1967.

[26] D. Henry, Geometric Theory of Semilinear Parabolic Equations, vol. 840 of Lecture Notes in Mathematics, Springer, Berlin, Germany, 1981.

[27] Y. H. Du, "Fixed points of increasing operators in ordered Banach spaces and applications," Applicable Analysis, vol. 38, no. 1-2, pp. 1-20, 1990.

[28] A. Pazy, Semigroups of Linear Operators and Applications to Partial Differential Equations, vol. 44 of Applied Mathematical Sciences, Springer, New York, NY, USA, 1983.

[29] J. Liang, J. H. Liu, and T.-J. Xiao, "Nonlocal problems for integrodifferential equations," Dynamics of Continuous, Discrete \& Impulsive Systems. Series A, vol. 15, no. 6, pp. 815-824, 2008. 


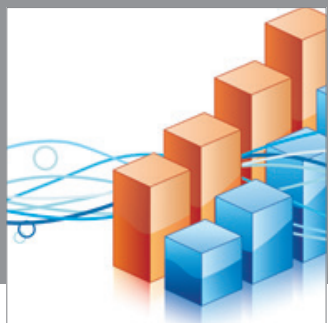

Advances in

Operations Research

mansans

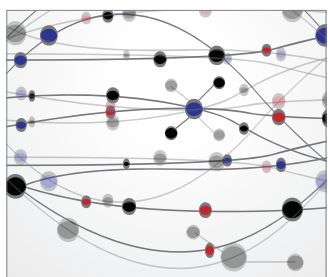

The Scientific World Journal
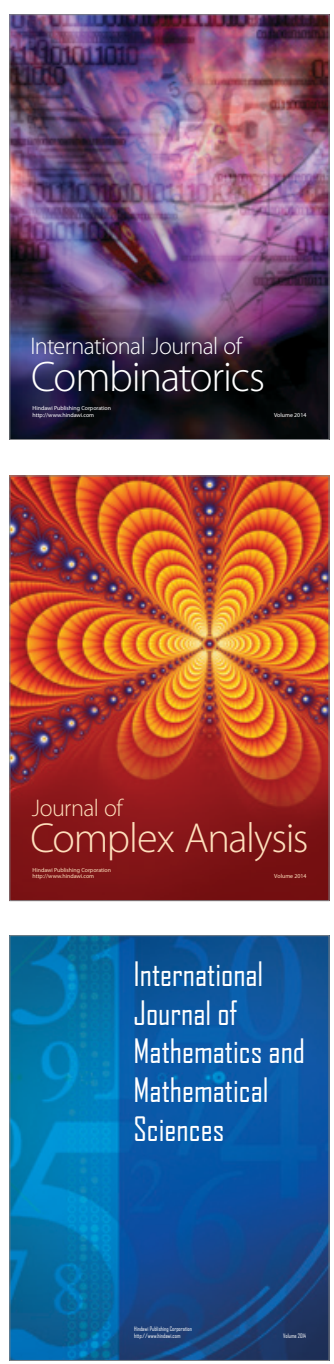
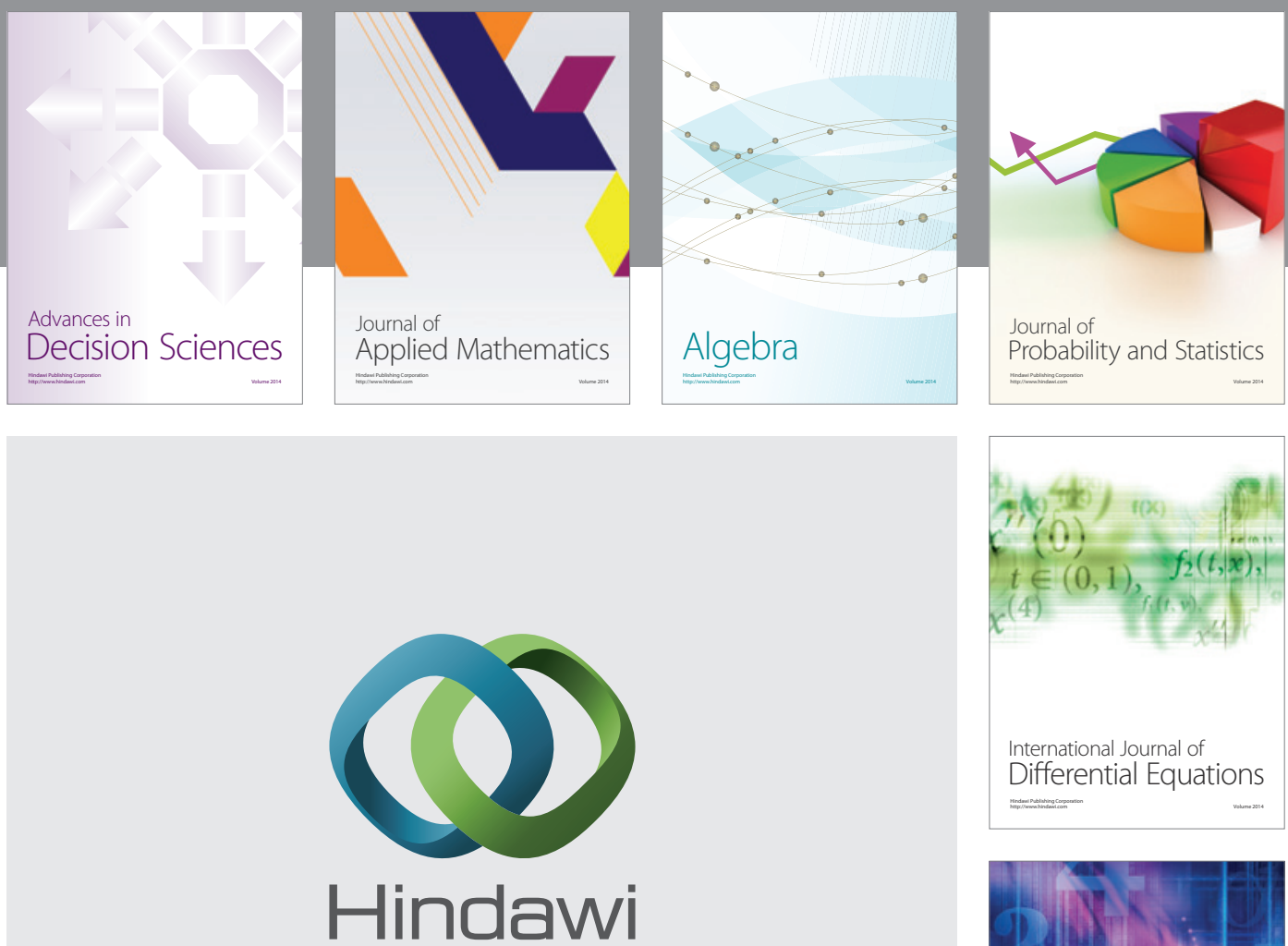

Submit your manuscripts at http://www.hindawi.com
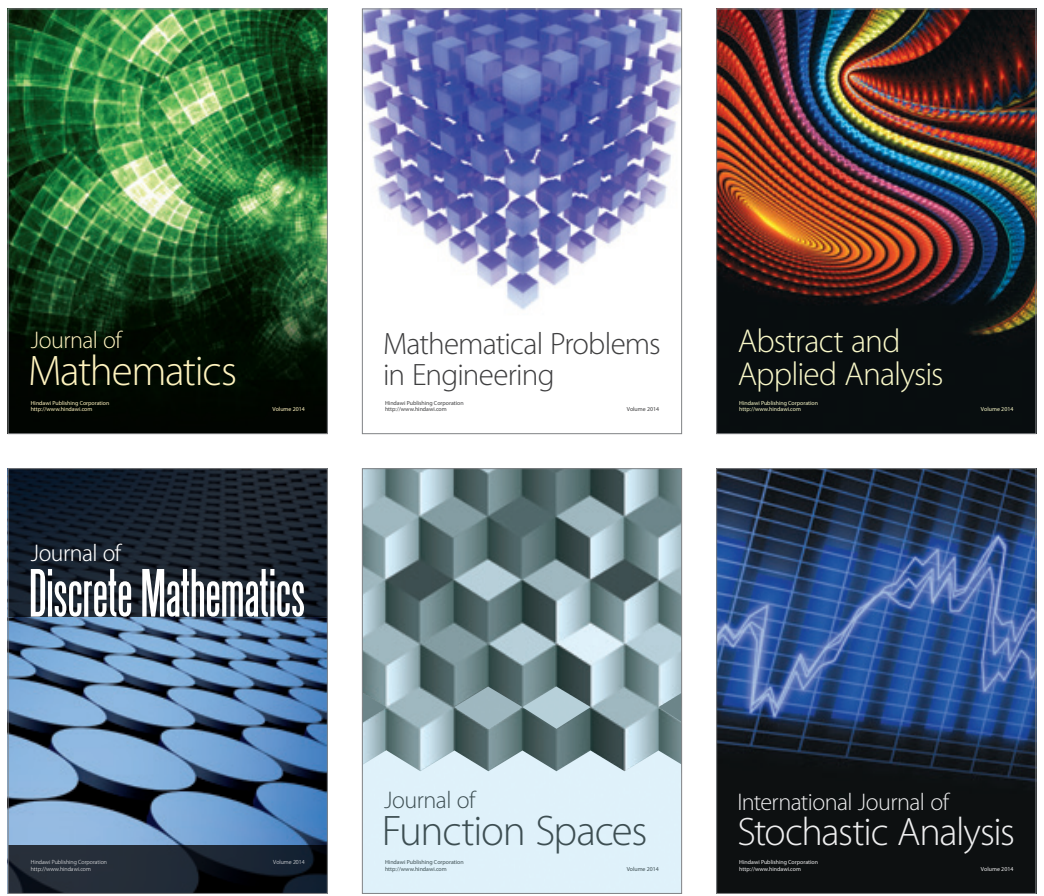

Journal of

Function Spaces

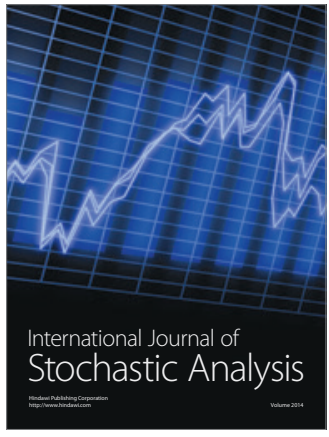

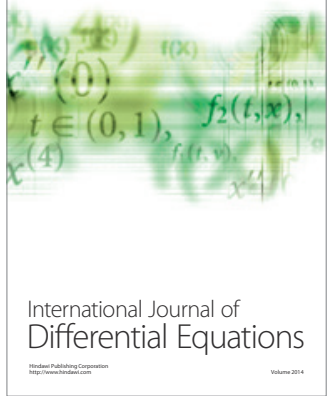
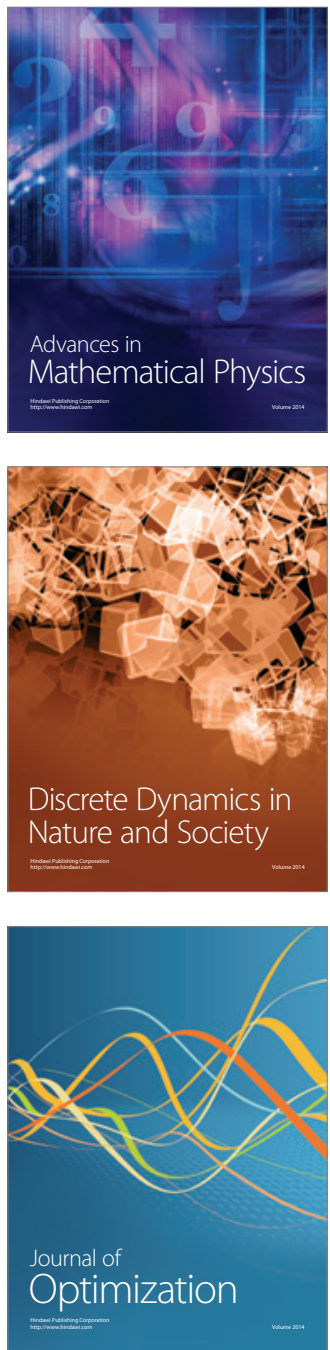